\title{
Abhandlung
}

Prof. Dr. Sabine Gless* und Prof. Dr. Ruth Janal

\section{Hochautomatisiertes und autonomes Autofahren - Risiko und rechtliche Verantwortung}

DOI 10.1515/juru-2016-0072

\section{A. Einleitung}

Das Kraftfahrzeug hat im vergangenen Jahrhundert als "Kutsche ohne Pferd" unser Straßenbild und die Automobilität des Einzelnen revolutioniert; es hat unsere Städte und Landschaften ebenso verändert wie unsere Wirtschaft und unser Recht. Heute löst die Vision einer »Kutsche ohne Kutscher« neue Überlegungen zu Risiko und rechtlicher Verantwortung aus. Dies geschieht vor dem Hintergrund einer zunehmenden Automatisierung von Fahrvorgängen, welche allmählich die Kontrolle der Autofahrt vom Fahrer zum Auto verschiebt. ${ }^{1}$ Wird diese anvisierte $»$ Revolution der Automobilität $\aleph^{2}$ Realität, werden sich unsere Städte und Landschaften, unsere Wirtschaft und auch unser Rechtsdenken erneut verändern. ${ }^{3}$

Gegenstand der folgenden Abhandlung ist die Frage, ob das hochautomatisierte Autofahren bzw. das autonome Autofahren Anlass gibt, traditionelle Instrumente der Verantwortungszuweisung für Schäden aus Verkehrsunfällen zu ändern oder zu ergänzen, um die Haftungsfrage im Straßenverkehr adäquat zu regeln. Noch sind viele rechtstatsächliche Rahmenbedingungen offen, etwa ob in den

1 Grundlegend zu diesem Trend in verschiedenen Bereichen: Beck, JR 2016, 225 (227 ff.).

$2 \mathrm{Zu}$ diesem Thema fand am 23. September 2015 eine Fachtagung der Daimler AG unter dem Titel »Autonomes Fahren im Spiegel von Recht und Ethik. Von der Kutsche ohne Pferd zur Kutsche ohne Kutscher« in Frankfurt a.M. statt. Die Überlegungen zur strafrechtlichen Haftung wurden dort von Sabine Gless vorgetragen.

3 »Wir fahren Auto-Auto«, Die Zeit vom 3. September 2015, S. 29f.; Both/Weber, Hands-Free Driving? Automatisiertes Fahren und Mensch-Maschine Interaktion, in: Hilgendorf (Hrsg.), Robotik im Kontext von Recht und Moral, Robotik und Recht Band 3, Baden-Baden 2014, S. $171 \mathrm{ff}$.

*Kontaktperson: Sabine Gless, Universität Basel, Juristische Fakultät, https://ins.unibas.ch.

Ruth Janal, Freie Universität Berlin, Fachbereich Rechtswissenschaft, www.jura.ju-berlin.de. kommenden Jahren Autos auf öffentlichen Straßen tatsächlich vollständig ohne Fahrer auskommen werden ob die künftige Autotechnik weitgehend auf »machine learning « basieren wird oder auf klassischer Programmierung. Klar ist bereits, dass Autounfälle durch die Automatisierung des Verkehrs erheblich verringert, aber nicht ganz ausgeschlossen werden können. ${ }^{4}$ Wer künftig für einen Unfall als verantwortlich angesehen wird, dürfte unter anderem von Technik und Vermarktung abhängen, ${ }^{5}$ und lässt sich ohne Kenntnis der künftigen technischen und wirtschaftlichen Eckpunkte nicht endgültig vorhersagen. Gleichwohl müssen Abwägungen zur rechtlichen Verantwortung bereits im Grundsatz geklärt sein, bevor autonome Autos auf öffentlichen Straßen fahren, damit für alle fassbar ist, wer unter welchen Voraussetzungen in der Verantwortung steht.

\section{B. Players on the Field}

Nach einer langen Zeit, in der die Kutsche ohne Pferd unsere Realität geprägt hat, aber Rechtsdenken aus der Tierhalterhaftung fortgeführt wurde, scheint nun die Zeit der Kutsche ohne Kutscher angebrochen. Es stellt sich damit die Frage, ob traditionelle Haftungskonzepte beibehalten werden können. ${ }^{6}$

$4 \mathrm{Zu}$ verschiedenen Zwischenfällen in jüngerer Zeit vgl. 〈http://www. reuters.com/article/us-google-selfdrivingcar-idUSKCNOW22DG〉 vom 29. Februar 2016; Lüssi, Tesla-Fahrer baut Unfall auf A1 - wegen Autopilot, 〈http://www.20min.ch/schweiz/news/story/30228076 vom 31. Mai 2016; Kuri, Tödlicher Tesla-Unfall: Autopilot hielt Lastwagen-Anhänger für hohes Schild, http://heise.de/-3253449〉 vom 4. Juli 2016.

5 Nach Industrieangaben soll der Aufpreis für autonome Steuerungssysteme bei bis zu 4 500EUR liegen, vgl. Doll, Warum selbstfahrende Autos so teuer sein werden, 〈http://www.welt.de/wirtschaft/article145015200/Warum-selbstfahrende-Autos-so-teuer-sein-werden. html vom 10. August 2015.

6 Vgl. BGHZ 13, 351; aus der schweizerischen Rechtsprechung: BGE 129 III $102 \mathrm{ff}$. (103), E. 2.1; BGE 117 II $609 \mathrm{ff}$. (612), E. 3. 
Schalten Autofahrer künftig im Stau auf Auto-Pilot, ${ }^{7}$ um die erwartete Verspätung per Textnachricht durchzugeben, dürfte sich die Anzahl von Auffahrunfällen im Stop-and-go-Verkehr deutlich verringern, weil sich Fahrer nicht mehr gleichzeitig dem Fahren und der fahrfremden Tätigkeit widmen werden. Aber Autofahren bleibt gefährlich, Unfälle weiter möglich. Knallt es künftig im Stop-andgo - etwa wenn das autonome Fahrzeug in eine befahrene Spur wechselt oder ein am linken Fahrbahnrand abgestelltes Fahrzeug nicht erkennt ${ }^{8}$ - dann fällt der Blick der Insassen des angefahrenen Autos zunächst auf das aufgefahrene Auto, dann auf den darin sitzenden Fahrer (der jedoch Unschuld beteuernd die Hände hebt, die er gar nicht am Lenkrad hatte); dann rückt der Fokus auf den Fahrzeughalter, zunehmend aber auf den Hersteller des Fahrzeugs oder das Unternehmen, welches die Software liefert bzw. den für dieses arbeitenden Techniker und Programmierer. Man könnte einen Schuldigen aber auch beim Datenlieferanten suchen, der Informationen über Straßenbelag oder Witterungsverhältnisse zur Verfügung stellt; ${ }^{9}$ vielleicht denkt man sogar an Hacker. ${ }^{10}$

Gerade Zeiten grundlegender technologischer Innovation zeigen, dass jede Haftung auf gesellschaftlicher $\mathrm{Zu}$ schreibung von Risikozuständigkeit basiert. Beispielhaft dafür ist die Weiterentwicklung zivilrechtlicher Haftung: Als der Motor das Pferd ablöste, war dies Anlass für die Einführung der verschuldensunabhängigen Haftung des Fahrzeughalters. Wenn bei der autonomen Autofahrt künftig auch der Kutscher durch eine Maschine ersetzt wird,

7 Derzeit steht einer solchen Handhabung das Zulassungsrecht entgegen, vgl. § 3 FZV. Völkerrechtliche Rahmenbedingungen setzt Art. 8 Abs. 5, 13 Abs. 1 S. 1 des Wiener Übereinkommens über den Straßenverkehr von 1968, wonach ein Fahrzeugführer sein Fahrzeug ständig »beherrschen« muss. Ein Änderungsvorschlag für das Übereinkommen, der autonome Assistenzsysteme berücksichtigt, liegt bereits auf dem Tisch, vgl. United Nations Economic Commission for Europe, Consistency between the 1968 Convention on Road Traffic and Vehicle Technical Regulations ECE/TRANS/WP.1/2014/1: some issues to be considered, März 2014, http://www.unece.org/fileadmin/DAM/trans/doc/2014/wp1/ECE-TRANS-WP1-Presentation-4e. pdf`., hierzu näher Lutz, NJW 2015, 119 (122ff.).

8 Shepardson, Google says it bears 'some responsibility' after selfdriving car hit bus, «http://www.reuters.com/article/us-google-selfdrivingcar-idUSKCNOW22DG〉 vom 29. Februar 2016; Lüssi, Tesla-Fahrer baut Unfall auf A1 - wegen Autopilot, http://www.20min.ch/ schweiz/news/story/30228076 vom 31. Mai 2016.

9 Siehe zum Informationsfluss aus der Cloud sowie zur Car-to-CarKommunikation Jourdan/Matschi, NZV 2015, 26 (27ff.).

10 Siehe nur Greenberg, Hackers Remotely Kill a Jeep on the Highway - With Me in It, ‘http://www.wired.com/2015/07/hackersremotely-kill-jeep-highway> vom 21. Juli 2015. bietet es sich an, die obige Liste potentieller Verantwortlicher um eine weitere Figur zu erweitern: Die Figur des Betreibers, der anstelle des Fahrers die Verantwortung für den intellektuellen Akt des Fahrens übernimmt. Eine adäquate Definition dieser Figur fällt jedoch schwer, solange weder die einem autonomen Auto künftig zugrunde gelegte Technik, noch dessen Vermarktung konkret festgelegt sind.

Für die Zwecke dieser Abhandlung wird unter dem Begriff des Betreibers diejenige Person verstanden, die rechtlich und technisch die Verantwortung für die Funktionstüchtigkeit des Steuerungssystems, insbesondere den Bezug externer Daten sowie deren fortlaufende Aktualisierung und Verarbeitung übernimmt. Das könnte sowohl ein Automobilhersteller sein, der die Verantwortung für den Betrieb des Steuerungssystems bei sich behält, aber auch ein unabhängiges Software-Unternehmen, welches die Pflichten zum Betrieb des autonomen Fahrens übernommen hat. ${ }^{11}$ Neben dem Betreiber, der anstelle des Fahrers (zeitweise) die Kontrolle über den Fahrvorgang hat, bleibt weiterhin der Halter relevant, welcher das Fahrzeug für eigene Rechnung gebraucht bzw. die physische Verfügungsgewalt über den Gebrauch hat. Nicht unter den Begriff des Betreibers autonom fahrender Autos fallen nach der hier vorgeschlagenen Definition etwa bloße Wartungstechniker, welche punktuelle Pflichten zur Wartung autonomer Fahrzeuge und zur Aktualisieren der Software vertraglich übernehmen. Auch Unternehmen, die lediglich die jeweiligen Updates programmieren oder Datenlieferanten, die Informationen über Straßenbelag oder Witterungsverhältnisse fortlaufend in das System einspeisen, erscheinen grundsätzlich nur als Hilfspersonen derjenigen, die für den insgesamt ordnungsgemäßen Betrieb eines Fahrzeuges einstehen müssen. Vom Betreiber in diesem Sinne ebenfalls $\mathrm{zu}$ unterscheiden sind bloße Hersteller autonomer Fahrzeuge oder von Fahrerassistenzsystemen bzw. Steuerungssoftware, die nach Verkauf des aus ihrer Sicht fertigen Produktes keine weiter gehenden Pflichten übernehmen.

Die Figur des Betreibers erscheint notwendig, um die faktische Verantwortungsverlagerung beim autonomen

11 Eine erste Kooperation zwischen Automobilhersteller und Digitalkonzern wurde zwischen Google und Fiat Chrysler vereinbart, siehe Fromm, Fiat Chrysler und Google - ein kalkulierter Tabubruch, 〈http://www.sueddeutsche.de/wirtschaft/kooperation-fiat-chryslerund-google-ein-kalkulierter-tabubruch-1.298077> vom 5. Mai 2016. BMW, mobileye und Intel wollen eine offene Plattform schaffen, die auch anderen Fahrzeugherstellern offen steht, vgl. Pressemitteilung vom 1. Juli 2016, https://newsroom.intel.com». 
Fahren adäquat zu erfassen: Denn mit dem automatisierten Fahren verschiebt sich die Kontrolle über den Fahrvorgang vom Fahrer auf denjenigen, der Herrschaft über die Datensteuerung hat. Ob gleichzeitig mit einer Haftungsbegründung von Betreibern Fahrzeughalter aus der Verantwortung entlassen werden könnten, weil bzw. wenn mit dem automatisierten Betrieb ihre Kontrollmöglichkeiten verloren gehen, bleibt im Folgenden zu klären. Es liegt insoweit auf der Hand, dass sich die Haftungskonzepte im Strafrecht nicht zwingend parallel zum Zivilrecht entwickeln müssen. ${ }^{12}$ Denn das Zivilrecht kennt die Haftung für vermutetes Verschulden sowie die Gefährdungshaftung, ${ }^{13}$ während ein strafrechtlicher Vorwurf einen Menschen nur bei erwiesenem persönlichem Fehlverhalten trifft. $^{14}$

\section{Bestehende Haftungskonzepte}

Verantwortung für Schädigungen durch Verkehrsunfälle tragen nach klassischen Haftungskonzepten drei Personengruppen: Autofahrer, Produzenten und Halter.

12 Hilgendorf, Recht und autonome Maschinen - ein Problemaufriß, in: Das Recht vor den Herausforderungen der modernen Technik, Robotik und Recht Band 4, Baden-Baden 2015, S. 11 ff. (16); derselbe, Teilautonome Fahrzeuge: Verfassungsrechtliche Vorgaben und rechtspolitische Herausforderungen, in: Hilgendorf/Hötitzsch/Lutz (Hrsg.), Rechtliche Aspekte automatisierter Fahrzeuge, Beiträge zur 2. Würzburger Tagung zum Technikrecht im Oktober 2014, Robotik und Recht Band 5, Baden-Baden 2015, S. $15 \mathrm{ff}$. (25ff.); Beck, JR 2009, S. $225 \mathrm{ff}$.

13 Frister, Strafrecht Allgemeiner Teil, 7. Aufl., München 2015, S. $29 \mathrm{ff}$. Das schweizerische Recht kennt mit Art. 6 OBG (Ordnungsbußengesetz, SR 741.03) den Fremdkörper einer verschuldensunabhängigen strafrechtlichen Halterhaftung; kritisch etwa Wohlers, Straßenverkehr 1/2015, S. 5 ff. (14f.); aus deutscher Sicht erscheint eine solche Regelung nicht denkbar: Milke, NZV 2010, S. 17 ff. (19f.); vgl. aber § 25a Abs. 1 StVG, der immerhin eine Auflage der Verfahrenskosten an den Halter vorsieht, wenn ein Halt- oder Parkverstoß begangen wurde und der Fahrer nicht ermittelt werden kann oder dessen Ermittlung einen unangemessenen Aufwand erfordern würde. 14 Frister, Strafrecht Allgemeiner Teil, 7. Aufl., München 2015, S. 29; Kindhäuser, Strafrecht Allgemeiner Teil, 7. Aufl., Baden-Baden 2015, $\S 21 \mathrm{~N} 5$ ff.; Kuhlen, Strafrechtliche Produkthaftung, in: Achenbach/ Ransiek/Rönnau, Handbuch Wirtschaftsstrafrecht, 4. Auflage, Heidelberg 2015, S. 124ff. Aufgeweicht wird diese Einschränkung allerdings durch die Figur des Übernahmeverschuldens, vgl. dazu Kindhäuser/Neumann/Paeffgen, NK StGB, 4. Auflage 2013, Vor § 13 N 160; Stratenwerth, Schweizerisches Strafrecht, Allgemeiner Teil I, 4. Aufl., Bern 2011, § 14 N 29.

\section{Haftung von Autofahrern}

Eine straf- oder zivilrechtliche Verschuldenshaftung des Autofahrers kommt - auch beim Einsatz automatisierter Systeme - nur in Betracht, wenn man ihm jedenfalls Fahrlässigkeit vorwerfen kann, also dass er (a) den tatbestandsrelevanten Erfolg (bspw. eine Körperverletzung) hätte voraussehen können und (b) nicht die notwendige Sorgfalt aufgewandt hat, um diesen Erfolg zu vermeiden.

Autofahrer wollen Autopiloten für den Stop-and-goVerkehr, weil sie fahrfremden Tätigkeiten nachgehen können, während die Fahrassistenz das Fahrzeug sicher und bequem bewegt. Gleichzeitig wissen sie, dass - wie bei jeder Technologie - ein gewisses Restrisiko bleibt, selbst wenn intelligente Assistenzen, anders als traditionelle Maschinerie, ihre Reaktionen ständig optimieren. Denn auch wenn Staupiloten selbständig Umgebungsinformationen auswerten, darauf präzise und unermüdlich reagieren und nebenbei noch ihr Fahrverhalten perfektionieren, wird sich die Zahl der Auffahrunfälle nicht auf null reduzieren. Denn Sensoren können bspw. ganz profan verschmutzt sein $^{15}$ oder ein bestimmtes Farbspektrum schlicht nicht richtig erkennen. ${ }^{16}$ Dieses allgemeine Risiko ist jedem $\mathrm{Au}$ tofahrer bekannt; das abstrakte Wissen genügt jedoch nicht für einen persönlichen Vorwurf, wenn es tatsächlich zum Unfall kommt. Den Fahrer kann ein Vorwurf vielmehr nur dann treffen, wenn er entweder selbst die Fehlerquelle ist oder eine maschinelle Fehlerquelle erkannt hat bzw. hätte erkennen können und müssen. Dies ist etwa der Fall, wenn der Fahrer auf ein zurückrufendes Signal, z.B. in Form von Blinkzeichen und/oder Warntönen, nicht reagiert oder ein Auto fährt, dessen Autopilot nach Herstellerangaben noch der Überwachung bedarf oder den er bereits als gestört erkannt hat. Daneben kommt natürlich ein schuldhafter Eingriff des Fahrers in das autonome Steuerungssystem durch Custom-ROMs in Betracht, d.h. durch Software von Drittanbietern, mit denen die Fähigkeiten des Produkts erweitert oder die herstellerseitig vorgesehenen Betriebsbeschränkungen umgangen werden können. ${ }^{17}$

15 Zur Blockade eines Radars durch ein großes Insekt siehe Smith, A giant moth managed to knock out a Tesla car's Autopilot system, http://www.techinsider.io/tesla-autopilot-knocked-out-by-moth-

2016-5> vom 11. Mai 2016.

16 Kuri, Tödlicher Tesla-Unfall: Autopilot hielt Lastwagen-Anhänger für hohes Schild, «http://heise.de/-3253449〉 vom 4. Juli. 2016.

17 Verbreitet sind derartige Custom-ROMs insbesondere für Smartphones, siehe näher zum Begriff ^https://de.wikipedia.org/wiki/Custom-ROM〉 vom 1. Juni 2016. Zu Optionen, das HMI fahrerseitig innerhalb gesetzlich zulässiger Grenzen zu personalisieren siehe Jourdan/ Matschi, NZV 2015, 26 (28). 
Die Fahrlässigkeitshaftung hängt immer von den konkreten Umständen im Einzelfall ab. Wenn sich das autonome Fahren in Zukunft in bestimmten Verkehrssituationen durchgesetzt hat, ist durchaus denkbar, dass sich der Fahrer einem Fahrlässigkeitsvorwurf ausgesetzt sieht, weil er selbst fährt, anstatt einen maschinellen Autopiloten zu nutzen. ${ }^{18}$

\section{Haftung von Herstellern und Betreibern}

Werden Fahrer künftig durch automatisierte Vorgänge ersetzt, müssen sie aus der Verantwortung genommen werden. Das steht hinter der Forderung des Goslarer Verkehrsgerichtstags 2015, ab »dem hochautomatisierten Fahrbetrieb [... den] Fahrer bei bestimmungsgemäßem Gebrauch von Sanktionen und der Fahrerhaftung frei zu stellen «. ${ }^{19}$ Käme der Gesetzgeber der Forderung des Goslaer Verkehrsgerichtstags 2015 nach, so rückten wohl Fahrzeughersteller sowie Betreiber als Verantwortliche für den intellektuellen Akt des Fahrens in die Verantwortungsstellung.

\section{Strafrechtliche Produktehaftung}

$\mathrm{Zu}$ Recht sprechen die Teilnehmer des Verkehrsgerichtstags mögliche Veränderungen einer strafrechtlichen Haftung an, wenn künftig automatisierte Vorgänge zu einem Schadenseintritt führen. Dahinter steht die Vorstellung, dass Körperverletzung oder gar Tötungen im Strassenverkehr nicht generell straflos bleiben können, die strafrechtliche Haftung aber immer einen individuellen Schuldvorwurf voraussetzt. Könnten Ingenieure, Programmierer oder Betreiber als neue Verantwortliche für das hochautomatisierte bzw. autonome Fahren insoweit an die Stelle des Fahrers treten? Auch ihnen kann fahrlässiges Handeln (nur) vorgeworfen werden, wenn sie sich sorgfaltswidrig verhalten; das heisst, wenn sie jene Vorsicht nicht beachten, zu der sie nach den Umständen und nach ihren persönlichen Verhältnissen verpflichtet sind. ${ }^{20}$ Kann dies

18 So bereits zu derzeit verfügbaren Assistenzsystemen Vogt, NZV 2003, 153 (157); anders Jourdan/Matschi, NZV 2015, 26 (27): Es gehe nicht darum, den Fahrer zu entmündigen.

19 Abrufbar unter: www.gdv.de; dazu Hilgendorf, Automatisiertes Fahren und Recht, in: Deutscher Verkehrsgerichtstag (53., 2015, Goslar). 2015, 55 (64).

20 Frister, Strafrecht Allgemeiner Teil, 7. Aufl., München 2015, S. 162ff.; Stratenwerth/Kuhlen, Strafrecht Allgemeiner Teil, 6. Aufl., München 2011, § 15 N $15 \mathrm{ff}$ bereits dann der Fall sein, wenn sie automatisierte Fahrassistenzen trotz des Wissens um ein Restrisiko in den Verkehr bringen und es dann tatsächlich zum Unfall kommt?

\section{a. Vorhersehbarkeit des Erfolgseintritts}

Im Strafrecht geht man davon aus, dass die Vorhersehbarkeit einer möglichen Schadensverursachung mehr oder weniger zwangsläufig auf eine Sorgfaltspflichtverletzung schließen lässt. ${ }^{21}$ Doch genau das erscheint beim Einsatz sog. intelligenter Systeme zweifelhaft. Denn auch die Entwickler und Betreiber des besten Staupiloten können nicht mit 100-prozentiger Sicherheit vorhersagen, wie das Auto in einer bestimmten Verkehrssituation durch die Verknüpfung der unterschiedlichen Datenverarbeitungsvorgänge reagieren wird - und zwar nicht nur dann, wenn es auf eine Situation trifft, für die keine Reaktion vorprogrammiert wurde, sondern insbesondere dann, wenn es aus den bisherigen Erfahrungen lernt. So ist beispielsweise vorstellbar, dass ein durch umfangreiche Testreihen geprüfter Umgebungssensor ein besonderes Farbspektrum eines Heckspoilers eines vorausfahrenden Autos fehlerhaft verarbeitet und das auf dieser Grundlage automatisiert fahrende Auto deshalb davon ausgeht, es habe freie Fahrt oder jedenfalls einen größeren Abstand zum vorausfahrenden Auto als das in Wahrheit der Fall ist. Dürften sich Produzenten oder Betreiber in diesem Fall darauf berufen, sie hätten dieses Fehlverhalten nicht vorhersehen können, da das Auto aufgenommene Daten in Testläufen stets korrekt verarbeitet habe und müssten schon deshalb straffrei ausgehen? Oder müsste man den genau umgekehrten Schluss ziehen: Dass nämlich diejenigen, die intelligente Fahrzeuge auf die Straße bringen, einfach mit »allem« rechnen müssen, dass also jede Art von Schädigung prinzipiell vorhersehbar ist. ${ }^{22}$ Folgte man der erstgenannten Argumentation, käme das fast einer Art carte blanche für Produzenten und Betreiber automatisiert fahrender Autos gleich. Folgt man der zweitgenannten Argumentation, ist Fahrlässigkeitshaftung grundsätzlich möglich, wenn einer oder mehreren an der Herstellung beteiligten Person(en) ein sorgfaltswidriges Verhalten vorgeworfen werden kann. ${ }^{23}$ Es sind dann weitere Hürden zu überwinden: Es muss ein adäquater Sorgfaltsmaßstab for-

21 Vgl. etwa Duttge, in: Münchener Kommentar zum StGB, 2. Aufl. 2011, § 15 Rn. 121ff.

22 Gless/Weigend, ZStW 2014, S. $581 \mathrm{f}$.

23 Vgl. Sternberg-Lieben/Schuster, Kommentar Strafgesetzbuch, 29. Aufl., München 2014, N 216 zu § 15 StGB; Kuhlen, Strafrechtliche Produkthaftung, in: Achenbach/Ransiek/Rönnau, Handbuch Wirt- 
muliert und dessen Verletzung durch einen an der Produktionskette beteiligten Menschen nachgewiesen werden.

\section{b. Standard für Sorgfaltspflichten}

Zunächst werfen intelligente Fahrassistenzen ein neues Licht auf an sich altbekannte Probleme der Produkthaftung: Grundsätzlich gelten dort für die Herstellung und den Vertrieb potentiell gefährlicher Produkte besonders strenge Sorgfaltsregeln, ${ }^{24}$ die weitgehend aus dem Zivilrecht ins Strafrecht transferiert worden sind. ${ }^{25}$ Ein Hersteller darf nur solche Produkte auf den Markt bringen, deren Sicherheit durch ausreichende Tests überprüft worden ist und dem Stand von Wissenschaft und Technik entspricht. Wie diese Sorgfaltspflichtanforderungen für den Vertrieb autonomer Fahrzeuge zu konkretisieren sind, ist noch unklar. Ist es ausreichend, Halter und Fahrer darauf hinzuweisen, dass ein per Update aufgespielter Autopilot der Überwachung bedarf und der Fahrer deshalb die Hände am Steuer lassen solle? ${ }^{26}$ Genügt es, bei der Konzeption von deep learning-Programmen die erforderliche Sorgfalt einzuhalten oder müssen die Ergebnisse solcher Lernprozesse stets durch Testreihen evaluiert und das Verhalten der Nutzer überwacht werden? Wie ist der aktuelle Stand von Wissenschaft und Technik angesichts rasanter Entwicklungsfortschritte adäquat zu bestimmen?

Auch wenn sich für intelligente Autos noch keine festen Standards etabliert haben, folgt aus dem geltenden Produkthaftungsrecht grundsätzlich eine Beobachtungspflicht - etwa anhand von Rückmeldungen durch Ver-

schaftsstrafrecht, 2. Teil, 1. Kapitel, Rdn. 7, 4. Auflage, Heidelberg 2015.

24 Gless, recht 2013, S. 56; vgl. auch Sternberg-Lieben/Schuster, Kommentar Strafgesetzbuch, 29. Aufl., München 2014, N 216 zu § 15 StGB.

25 Grundlegend BGHSt 37, 106 (»Lederspray«). Zum Verhältnis von zivilrechtlicher und strafrechtlicher Produkthaftung Gless, recht 2013, 57 f.; Kuhlen, Strafrechtliche Produkthaftung, in: Achenbach/Ransiek/Rönnau, Handbuch Wirtschaftsstrafrecht, 2. Teil, 1. Kapitel, Rdn. 29, 4. Aufl., Heidelberg 2015; ders., JZ 1994, 1142; Schlutz, DStR 1994, 1811, 1814; Vogel, in: LK, § 15 StGB Rdn. 277-281. Zu der »Lederspray«-Entscheidung siehe Kuhlen, NStZ 1990, 566; Hilgendorf, NStZ 1994, 561.

26 So das Vorgehen von Tesla; siehe Schaal, Ärger für Teslas Autopiloten, 〈http://www.wiwo.de/unternehmen/auto/selbstfahren de-autos-aerger-fuer-teslas-autopiloten/12528826.html > vom 2. November 2015. Bei Fehlsteuerungen des Autopiloten weist Tesla jede Verantwortung ab, siehe Lüssi, Tesla-Fahrer baut Unfall auf A1 wegen Autopilot, «ttp://www.20min.ch/schweiz/news/story/302280 76) vom 31. Mai 2016; Kuri, Tödlicher Tesla-Unfall: Autopilot hielt Lastwagen-Anhänger für hohes Schild, 〈http://heise.de/-3253449〉 vom 4. Juli 2016. braucher. Zeigen sich unerwartete Schäden oder Risiken, so ist der Hersteller zur Warnung der Nutzer und nötigenfalls zum Rückruf des Produkts verpflichtet. ${ }^{27}$ Eine Marktzulassung entlastet also nicht. Doch ist der Nachweis einer strafrechtlich relevanten Sorgfaltspflichtverletzung in der Praxis schon wegen der arbeitsteiligen Organisation von Entwicklungs- und Produktionsprozessen, wie in der $\mathrm{Au}-$ tomobilindustrie heute bereits üblich, praktisch nicht leicht zu führen. ${ }^{28}$

\section{c. Haftungsfreistellung}

Die Forderung nach einer Haftungsfreistellung wird nicht nur vom Verkehrsgerichtstag zugunsten der Fahrer, sondern auch von Herstellern ins Spiel gebracht - unter anderem mit Blick auf das Zusammenwirken vieler Akteure. Eine allzu strenge Haftung erschiene aus Herstellersicht unbefriedigend, wenn innovative Produkte von der Allgemeinheit begrüßt würden, wie das in vielen Bereichen des hochautomatisierten Fahrens der Fall ist. Denn bei autonomen Autos - wie bei anderen intelligenten Systemen - korreliert der erhoffte große Nutzen zwangsläufig mit der Gefahr, dass die Menschen, die diese Technologien entwickeln, deren Verhalten weder vollständig voraussehen noch kontrollieren können. Wendete man die bisher üblichen, strengen Haftungsgrundsätze ohne weiteres auf intelligente Fahrsysteme an, dann müsste die drohende strafrechtliche Haftung wohl dazu führen, dass Herstellung und Vertrieb bestimmter Technologien schlicht unterbleiben müssten. Diese Form der Innovation müsste verboten werden, selbst wenn eine Mehrheit der Gesellschaft der Ansicht wäre, dass sie das Leben sicherer und bequemer machen könnte. ${ }^{29}$

Wollte man diese Konsequenz vermeiden, müsste man für eine Reduktion des Haftungsrisikos im Strafrecht für Hersteller optieren: Entweder, indem man die gebotene Sorgfalt entsprechend den Besonderheiten intelligenter Systeme definiert oder, indem man eine Haftungsfreistellung auch im Strafrecht in allen Fällen akzeptiert, in denen der Stand von Wissenschaft und Technik eingehalten wird und man von einer Innovation einen besonderen, gesamtgesellschaftlichen Nutzen erhofft. ${ }^{30}$ Den Einbau automati-

27 Kuhlen, Strafrechtliche Produkthaftung, in: Achenbach/Ransiek/ Rönnau, Handbuch Wirtschaftsstrafrecht, 2. Teil, 1. Kapitel, Rdn. 40, 4. Aufl., Heidelberg 2015; Weigend, in: LK, 12. Aufl. 2007, § 13 Rdn. $53 \mathrm{f}$.

28 Vgl. Lutz, NJW 2015, S. 121; Sternberg-Lieben/Schuster, in Schönke/Schröder, StGB (29. Aufl.), § 15 Rn. 217.

29 Vgl. Gless/Weigend, ZStW 2014, S. 582ff.

30 Vgl. Gless/Weigend, ZStW 2014, S. $583 \mathrm{ff}$. 
sierter Technologie in ein Auto könnte man dann als »erlaubtes Risiko« qualifizieren, das schon von vornherein keine Sorgfaltspflichtverletzung darstellt oder jedenfalls gerechtfertigt ist, wenn alle relevanten Sorgfaltsregeln eingehalten werden. Man würde dann letztlich in der Haftungssituation ein gesamtgesellschaftliches Anliegen über das Strafinteresse stellen. ${ }^{31}$ Damit tut sich das Strafrecht schwer. ${ }^{32}$ Skepsis werden einem solchen Ansatz all diejenigen entgegen bringen, die glauben, dass gerade ein greifbares strafrechtliches Haftungsrisiko bessere, weil sicherere Innovationen begünstigt, weil Produzenten aus Angst vor strafrechtlicher Haftung besonders sorgfältig arbeiten. ${ }^{33}$ Auch verletzte Personen, Angehörige - und selbst die Innovationen an sich begrüßende Allgemeinheit könnten Mühe haben, zu akzeptieren, dass man ausschließlich auf eine zivilrechtliche Haftung zurück greift und gleichzeitig Fahrer, Hersteller und »Betreiber« eines automatisiert fahrenden Autos von einem strafrechtlichen Vorwurf freistellt. ${ }^{34}$

Das gilt insbesondere, wenn Verletzte beim Verdacht der Verletzung des Sorgfaltsstandards ohnehin noch die Hürden des Nachweises der im Einzelfall verletzten Sorgfaltspflicht überwinden müssen. Hier fehlt in Deutschland $\left(\right.$ noch $\left.^{35}\right)$ eine Behelfskonstruktion auf der Grundlage einer Unternehmensstrafbarkeit durch erleichterte strafrechtliche Verantwortungszuweisung an ein Kollektiv, wie man es in anderen Staaten kennt, etwa in der Schweiz durch die subsidiäre Unternehmensstrafbarkeit nach Art. 102 Abs. 1 des schweizerischen Strafgesetzbuches. ${ }^{36}$

31 Gless/Weigend, ZStW 2014, S. 583 f., 586 ff., 590f.; Sternberg-Lieben/Schuster, in Schönke/Schröder, StGB (29. Aufl.), § 15 Rn. 144 ff.; LK-Rönnau vor $\S 32 \mathrm{Rn}$. $53 \mathrm{ff}$.

32 Vgl. speziell zur Haftung bei Unfällen im Straßenverkehr: Nehm ZRP 2012, $70 \mathrm{ff}$.

33 So z.B. Spindler, CR 2015, 766 (774) zur zivilrechtlichen Verantwortung.

34 Zum Potenzial automatisierter Fahrzeuge im Hinblick auf die Reduktion von Verkehrstoten: Lutz, NJW 2015, S. 119; Hilgendorf, Recht und autonome Maschinen - ein Problemaufriß, in: Das Recht vor den Herausforderungen der modernen Technik, Robotik und Recht Band 4, Baden-Baden 2015, S. $11 \mathrm{ff}$.

35 Vgl. zur Unternehmenshaftung nach $\S 30$ OWiG: Beulke/Moosmayer, CCZ 2014, S. 146ff.; Sternberg-Lieben/Schuster, in Schönke/ Schröder, StGB (29. Aufl.), § 15 Rn. 217; Kuhlen, Strafrechtliche Produkthaftung, in: Achenbach/Ransiek/Rönnau, Handbuch Wirtschaftsstrafrecht, 2. Teil, 1. Kapitel, Rn. $5 \mathrm{ff}$.

36 StGB, SR 311.0 〈ttps://www.admin.ch〉. Vgl. Stratenwerth, Schweizerisches Strafrecht, Allgemeiner Teil I, 4. Aufl., Bern 2011, $\S 13$ N 181 ff.; Niggli/Gfeller, Basler Kommentar Strafrecht I, N 52ff. zu Art. 102 StGB.

\section{d. Innovation birgt Risiken}

Doch unabhängig von jeder Haftungsausgestaltung gilt, dass Innovation Risiko bedeutet. Häufig gibt erst der Schaden Anlass, etwas, was mit großer Euphorie begrüßt und rechtmäßig auf den Markt gelangt ist, aufgrund praktischer Erkenntnis zu verbessern. Weil die Allgemeinheit einem Risiko ausgesetzt wird, stehen Produzenten strafrechtlich in der Pflicht der Produktbeobachtung, Warnung und Sicherung. Mit einer behördlichen Zulassung, etwa mit der Testerlaubnis auf der Autobahn A9, entsteht genau diese Situation einer "schlafenden Ingerenz «. ${ }^{37}$ Sollte man feststellen, dass fahrerlose Autos mit der Verengung auf vier Spuren am Hermsdorfer Kreuz bei hohem Verkehr nicht zurechtkommen, müssen sie von der Straße genommen werden, egal wie die behördliche Genehmigung zum Testbetrieb aussieht. Denn ebenso wie es unfair erschiene, wenn die »Gesellschaft«, die den Nutzen aus der Innovation ziehen will, das unweigerlich mit ihr verbundene Risiko über das Strafrecht immer auf den Betreiber abwälzen könnte, wäre es nicht richtig, dass Betreiber sich prinzipiell auf ein »erlaubtes Risiko« berufen dürfen, obwohl sie erkennen, dass von ihren Autos in bestimmten Situationen eine konkrete Gefahr ausgeht. Wenn die »Kutsche ohne Kutscher« in unserem Straßenbild zur Normalität geworden ist, akzeptieren wir vielleicht eine rare ungeplante Fehlfunktion als allgemeines Lebensrisiko, so wie heute den für einen menschlichen Lenker unvermeidbaren Verkehrsunfall. ${ }^{38}$

Ein strafrechtsfreier Bereich für Verletzungen von Leib und Leben wird aber stets sehr klein sein. Denn selbst wenn die Gesellschaft bereit ist, bestimmte Risiken durch Computer gestützte Automatrix zu akzeptieren, wird sie beim Auto weiter Wert darauf legen, dass Gefahren durch sorgfältige Konstruktion und Programmierung, eingehende Tests und kontinuierliche Beobachtung so weit wie möglich minimiert werden. Die Haftung für fahrlässige und vorsätzliche Schädigungen kann nicht an sich ausgeschlossen werden..$^{39}$ Solange man Software-Agenten und Roboter nicht als bestrafungsfähig ansieht, verbleibt das Risiko strafrechtlicher Haftung bei den verantwortlichen Menschen. Andernfalls fände sich das Opfer einer schadensbringenden Aktion in der unbefriedigenden $\mathrm{Si}$

37 Im Sinne einer Verantwortlichkeit, die erst mit Erkennbarkeit der mit der Produktnutzung verbundenen Gefahren virulent wird, vgl. Gless, recht 2013, S. 60 f.

38 Vgl. etwa LG Görlitz, Urteil vom 28. April 2009, 5a Ns 130 Js 21475/ 07; Sternberg-Lieben/Schuster, in: Schönke/Schröder, StGB (29. Aufl.), § 15 Rn. 144.

39 Vgl. Gless/Weigend, ZStW 2014, S. 584f.; $590 f$. 
tuation einer Verantwortungsdiffusion ${ }^{40}$ zwischen Mensch und intelligenter Maschine, die jede strafrechtliche Ahndung selbst schwerster Schädigungen ausschlösse.

\section{Zivilrechtliche Produzentenhaftung}

Eine zivilrechtliche Haftung des Herstellers oder des Betreibers lässt sich leichter begründen. Denn einerseits bedarf es im Rahmen der zivilrechtlichen Produkthaftung nicht des Nachweises eines Verschuldens, andererseits können juristische Personen bzw. rechtsfähige Personengesellschaften zivilrechtlich zur Verantwortung gezogen werden.

\section{a. Vorteile der Produzentenhaftung}

Als Grundlage einer zivilrechtlichen Inanspruchnahme des Herstellers oder des Betreibers kommt einerseits die Produzentenhaftung nach $\S 823$ Abs. 1 BGB, andererseits die Produkthaftung gemäß§ 1 ProdHaftG in Betracht. An dieser Stelle wird nur die Produzentenhaftung erörtert: Erstens dürfte eine Inanspruchnahme des Betreibers der autonomen Steuerung nach dem Produkthaftungsgesetz ausscheiden, sofern dieser tatsächlich nur für den Betrieb verantwortlich zeichnet und nicht gleichfalls eine bewegliche Sache, d.h. Fahrzeug oder Steuerungssystem, herstellt (vgl. § 2 ProdHaftG). ${ }^{41}$ Zweitens ist auch bei der Inanspruchnahme des Fahrzeugherstellers die Produzentenhaftung für den Geschädigten regelmäßig vorteilhafter. ${ }^{42}$ Ausnah-

40 Beck JR 2009, 225 (227f.).

41 Ob Standardsoftware, die in einer Sache verkörpert ist und bei wertender Betrachtung Warencharakter aufweist, als Produkt i.S.v. $\S 2$ ProdHaftG anzusehen ist, ist umstritten, vgl. die umfassenden Nachweise bei Wagner in: MüKo-BGB, § 2 ProdHaftG Rn. 15f. Beim Betrieb eines autonom fahrenden Fahrzeugs dürfte ohnehin der Dienstleistungscharakter überwiegen, so dass ein Rückgriff auf das ProdHafG ausscheidet.

42 § 1 I 2 ProdHaftG schließt eine Haftung für Schäden an der gelieferten Sache selbst ebenso aus wie eine Haftung für Sachschäden im gewerblichen Verkehr. Nach § 1 Abs. 1 Nr. 5, § 3 Abs. 1 lit. c ProdHaft $G$ besteht keine Haftung für Sicherheitsrisiken, die zum Zeitpunkt des Inverkehrbringens des Fahrzeugs nicht erkennbar waren; für eine mangelnde Produktbeobachtung wird nicht gehaftet. § 11 ProdHaftG sieht eine Selbstbeteiligung i. H.v. 500EUR bei Sachschäden und § 10 ProdHaftG einen Haftungshöchstbetrag vor. Ob es möglich ist, einen Teilehersteller (Zulieferer der Steuerungseinheit) auf Ersatz der beschädigten Sache in Anspruch zu nehmen, ist umstritten, vgl. ablehnend OGH vom 3. Februar 1994 - 80b536/93 - zu §1 öst. PHG; Wagner in: MüKo-BGB, §1 ProdHaftG Rn. 11f.; befürwortend hingegen Kullmann, NZV 2002, 1 (9); Vogt, NZV 2003, 153 (158); Meyer/ Harland, CR 2007, 689 (694); Spindler, CR 2015, 766 (773). men bestehen nur dann, wenn der Geschädigte über $\S 4$ ProdHaft $\mathrm{G}$ gegen den Importeur vorgehen möchte oder den Produzent des Endprodukts für Fehler eines Teileherstellers zur Verantwortung ziehen möchte.

Die Produzentenhaftung trifft den Hersteller eines Produkts, sofern dieser durch das In-Verkehr-Bringen eines fehlerhaften Produkts bzw. eine nicht ausreichende nachfolgende Produktbeobachtung schuldhaft eine Verkehrssicherungspflicht verletzt hat und durch Verletzung eines der in $\S 823$ Abs. 1 BGB benannten Rechtsgüter ein Schaden verursacht wurde.

\section{b. Geschützter Personenkreis}

In den Schutzbereich der Produzentenhaftung sind neben dem Abnehmer auch Familienangehörige, Angestellte und unbeteiligte Dritte einbezogen..$^{43} \mathrm{Im}$ Falle eines durch ein autonom gesteuertes Auto verursachten Unfalls wäre grundsätzlich jeder am Unfall beteiligte Verkehrsteilnehmer zur Inanspruchnahme des Fahrzeugherstellers berechtigt.

Für den Geschädigten dürfte es aufgrund der schwierigen Beweislage im Rahmen der Produkt- und Produzentenhaftung allerdings auch künftig interessanter sein, seine Ansprüche gegenüber dem Fahrzeughalter geltend $\mathrm{zu}$ machen (hierzu näher infra III.2.). Die Inanspruchnahme des Produzenten oder Betreibers erfolgt somit primär im Wege des Rückgriffs durch den Kfz-Haftpflichtversicherer des Halters ${ }^{44}$ oder - sofern der Halter selbst verletzt wurde - durch Rückgriff der Krankenversicherung des Halters. Wurde das autonome Fahrzeug aufgrund einer Fehlsteuerung durch den Autopiloten beschädigt oder zerstört, dürfte auch der Fahrzeugeigentümer an der Inanspruchnahme des Produzenten bzw. Betreibers interessiert sein.

\section{c. Hersteller und Betreiber als Anspruchsgegner}

Bei der Ermittlung des passenden Anspruchsgegners ist zwischen Fehlern zu unterscheiden, die bereits zum Zeitpunkt der Fahrzeugauslieferung bestanden und Fehlern, die erst im Rahmen weiterer Updates in das System eingespielt wurden. Bezüglich bereits bei Systemauslieferung bestehenden Fehlern richtet sich der Anspruch aus § 823

43 Staudinger in Schulze, BGB, 8. Aufl. 2014, Rn. 158, 169; Teichmann in: Jauernig, BGB, 16. Aufl. 2015, § 823 Rn. 130.

$44 \mathrm{Zu}$ Forderungen, den Regress gegenüber Fahrzeugherstellern zu begrenzen siehe Kupferschmied, HAVE/REAS 2015, 356 (365); Albrecht, SVR 2005, 373, 375; Lutz NJW 2015, 119 (120f.); dem zu Recht entgegentretend Spindler, CR 2015, 766 (774); Lohmann/Rusch, HAVE/ REAS 2015, 349 (353). 
Abs. 1 BGB gegen den Autohersteller, der das Fahrzeug in den Verkehr gebracht hat. Der Fahrzeughersteller trägt grundsätzlich auch die Verantwortung für ein von Zulieferern bezogenes Steuerungssystem, jedoch nur, sofern der Produktfehler seinem Verantwortungs- und Organisationsbereich entsprungen ist und er sich nicht von der Verschuldensvermutung entlasten kann. ${ }^{45}$ Liegt die Entwicklung des Steuerungssystems allein beim Zulieferer, so besteht der Anspruch aus Produzentenhaftung nur gegenüber diesem. ${ }^{46}$ Hat der Zulieferer auf Anweisungen des Produzenten gehandelt oder hätte der Endhersteller den Fehler im Zuge eines Prüfverfahrens entdecken müssen, so sind beide als Hersteller anzusehen. ${ }^{47}$ Bei der Abgrenzung der Verantwortungsbereiche sind allerdings die in der $\mathrm{Au}$ tomobilindustrie typischen Qualitätssicherungsvereinbarungen zwischen Endhersteller und Zulieferer zu berücksichtigen. ${ }^{48}$

Ist der Fehler erst durch Produktupdates in das System eingespielt worden, kommt als Anspruchsgegner nur der Betreiber in Betracht, welcher zwar oftmals, aber nicht zwingend mit dem Hersteller oder Zulieferer identisch sein dürfte. Die Tatsache, dass der Betreiber keine bewegliche Sache herstellt, steht der Inanspruchnahme nach den Grundsätzen der Produzentenhaftung nicht entgegen, da diese im Kern eine Unternehmenshaftung ist. ${ }^{49}$

\section{d. Verkehrssicherungspflichten}

Hersteller wie Betreiber trifft die Pflicht zur sicheren Konzeption und Herstellung des Produkts, ${ }^{50}$ zur ordnungsgemäßen Instruktion der Produktnutzer sowie zur Pro-

45 Bauer, PHI 1989, 98 (101f.); Vogt, NVZ 2003, 153 (159); Meyer/ Harland, CR 2007, 689 (691, 693f.). Siehe allgemein OLG Schleswig, NJOZ 2013, 1366 (1367); Sprau in Palandt, § 823 Rn. 181. Zur Haftung für Verantwortungsbereiche nach $\S 823$ Abs. 2 bei Verstößen gegen $\S 3$ ProdSG siehe BGH VersR 2006, 710 (711); ferner EuGH NJW 2006, 204 (206) - Syuichi Yonemoto; Wagner in MüKo-BGB, § 823 Rn. 698.

46 Vgl. hierzu BGH NJW 1996, 2224 (2225ff.).

47 BGH NJW 1975, 1828; BGH VersR 1959, 104 (105); BGH NJW 1977, 379 (380); BGH NJW-RR 1990, 406; Bauer, PHI 1989, 98 (101f.); Lehmann, BB 1993, 1603 (1606f.); zur Abgrenzung nach der jeweiligen fachlichen Qualifikation BGH VersR 1990, 533; Foerste in Foerste/v. Westphalen, Produkthaftungshandbuch, § $25 \mathrm{Rn}$. $53 \mathrm{ff}$.

48 Näher Fuchs, JZ 1994, 533 (535f.); Wagner in MüKo-BGB, § 823 Rn. 629.

49 Ausführlich m.w. N. Wagner in MüKo-BGB, § 823 Rn. $623 \mathrm{f}$.

50 Bei autonomen Steuerungssystemen wird die Fehlerquelle regelmäßig aus der Programmierung resultieren, d.h. es wird ein Konstruktionsfehler vorliegen, vgl. Lutz, NJW 2015, 119 (120); Gomille, JZ 2016, 76 (77 f.); Meier/Wehlau, CR 1990, 95, 96; Reese, DStR 1994, 1121, 1123; Taeger, CR 1996, 257 (268). duktbeobachtung nach dem Inverkehrbringen. Angesichts des hohen Gefährdungspotentials des Straßenverkehrs für Leib und Leben der Verkehrsteilnehmer sind autonome Steuerungssysteme so zu entwickeln, dass sie zum Zeitpunkt des Inverkehrbringens dem neuesten Stand der Wissenschaft und Technik entsprechen..$^{51}$ Auch hier stellt sich das im Rahmen der strafrechtlichen Produkthaftung bereits angesprochene Problem einer adäquaten Bestimmung der Sorgfaltspflichten und des Stands von Wissenschaft und Technik. Den Hersteller bzw. Betreiber trifft zudem die Pflicht, die Steuerungssoftware gegen Manipulationsversuche Dritter (Hacks, Viren oder Würmer) zu sichern. ${ }^{52}$ Außerdem muss einem vorhersehbaren Fehlgebrauch durch den Nutzer vorgebeugt werden (z. B. deutlicher Rückruf bei erforderlicher menschlicher Steuerung, ggf. verbunden mit einem Abbremsen des Fahrzeugs, um einer versehentlichen Nichtübernahme durch den Fahrer vorzubeugen). Bedarf der Autopilot noch menschlicher Überwachung, so genügt es nicht, wenn der Fahrer auf diese Tatsache lediglich hingewiesen wird. Vielmehr bedarf es Prüfmechanismen, um sicherzustellen, dass der Fahrer die Hände am Steuer hat und die Aufmerksamkeit dem Straßenverkehr widmet.

Die Tatsache, dass Fehler bei der Entwicklung von Computerprogrammen nach einhelliger Auffassung unvermeidlich sind, ${ }^{53}$ führt nicht zu einer Haftungsfreistellung, sondern begründet höhere Qualitätssicherungsmaßnahmen und Produktbeobachtungspflichten. ${ }^{54}$ Dies gilt selbstverständlich auch für autonome Steuerungssysteme. ${ }^{55}$ Der Fahrzeughersteller bzw. Betreiber ist darüber hinaus zur Instruktion der Benutzer über mit dem autonomen Fahren verbundene Risiken verpflichtet. ${ }^{56}$ Besondere Instruktionspflichten dürften sich vor allem beim halbautomatisierten Fahren ergeben. Fahrer sind intensiv anzuleiten, in welchen Situationen das Fahrzeug autonom gesteuert werden kann, welche Verkehrssituationen eine menschliche Steuerung erfordern, wie ein Rückruf

$51 \mathrm{Zu}$ Sicherheitsstandards siehe BGHZ 181, 253 m.w. N.; OLG Hamm, VersR 11, 637.

52 Spindler, NJW 2004, 3145 (3146); Lohmann/Rusch, HAVE/REAS 2015, 349 (351); Gomille, JZ 2016, 77 (78).

53 Kupferschmied, HAVE/REAS 2015, 356 (363); Lehmann, NJW 1992, 1721 (1725).

54 Meier/Wehlau, CR 1990, 95 (96); Reese, DStR 1994, 1121 (1123f.); Taeger, CR 1996, 257 ff.; Horner/Kaulartz, CR 2016, 7 (11); Spindler in: Bamberger/Roth BGB, § 823 Rn. 564 m.w.N.

55 Spindler, CR 2015, 766 (768).

56 Meier/Wehlau, CR 1990, 95 (97); Reese, DStR 1994, 1121 (1123f.); Taeger, CR 1996, 257 (267); Spindler, CR 2015, 766 (770). 
des Fahrers erfolgt und wie sie sich für einen Rückruf bereitzuhalten haben. ${ }^{57}$

Besonders interessant mit Blick auf das autonome Fahren ist die Pflicht des Herstellers bzw. Betreibers zur Produktbeobachtung. Autonome Steuerungssysteme sind komplexe Neukonstruktionen mit erheblichem Schädigungspotential. ${ }^{58}$ Den Fahrzeughersteller trifft deshalb trotz ausreichender Produkttests die Pflicht, auch nach Inverkehrbringen die Funktionsweise des autonom gesteuerten Fahrzeugs und dessen Verwendung durch die Kunden aktiv zu beobachten ${ }^{59}$ und sich anhand aller zugänglichen Quellen über Entwicklungsfortschritte in Wissenschaft und Technik zu informieren. ${ }^{60}$ Gefahren können sich auch aus der gleichzeitigen Verwendung anderer, vermeintlich kompatibler Produkte ergeben ${ }^{61}$ (denkbar etwa: Funkstörungen durch gleichzeitig von den Insassen verwandte Geräte) oder durch Angriffe Dritter auf das Steuerungssystem. ${ }^{62} \mathrm{Um}$ seinen Verkehrssicherungspflichten hier nachzukommen, muss der Fahrzeughersteller bzw. der Betreiber den Nutzer über die erkannten Gefahren warnen. ${ }^{63}$ Bei Sicherheitslücken birgt eine Warnung freilich die Gefahr, dass die Information Dritten ermöglicht, die Schwachstelle auszunutzen. ${ }^{64}$ Hier dürfte dem Hersteller/Betreiber ein Ermessensspielraum zustehen, ob er eine öffentliche Warnung ausspricht oder versucht, die Sicherheit durch einen Patch wiederherzustellen, ohne die Lücke offenzulegen. ${ }^{65}$

\section{e. Rechtsfolgen}

Hat die schuldhafte Verletzung einer der oben genannten Verkehrssicherungspflichten zur Beeinträchtigung eines der in $\S 823$ Abs. 1 genannten Rechtsgüter geführt, ist der Hersteller bzw. der Betreiber zum Ersatz des entstandenen Schadens verpflichtet. $\mathrm{Zu}$ ersetzen sind insbesondere auch

57 Lutz/Tang/Lienkamp, NZV 2013, 57 (61); Jänich/Schrader/Reck, NZV 2015, 313 (317); Kupferschmied, HAVE/REAS 2015, 356 (362); zu Assistenzsystemen siehe bereits Vogt, NZV 2003, 153 (159).

58 Gomille, JZ 2016, 75 (80) m.w.N.

59 Hierbei sind die Rückmeldungen der vernetzten Fahrzeuge auszuwerten, vgl. zutreffend Droste, CCZ 2015, 105 (110); Gomille, JZ 2016, 75 (80); Horner/Kaulartz, CR 2016, 7 (12).

60 BGH NJW 1981, 1606 (1607f.); Vogt, NZV 2003, 153 (159); Gomille, JZ 2016, 75 (80).

61 BGH NJW 1987, 1009 (1010f.); für Einschränkungen der aktiven Produktbeobachtungspflicht mit Blick auf die Kompatibilität von Software plädiert Droste, CCZ 2015, 105 (107).

62 Hoeren, PHI 1989, 138 (141); Droste, CCZ 2015, 105 (109); kritisch Spindler, CR 2015, 766 (769f.).

63 BGH NJW 1981, 1606 (1607f.); BGH NJW 1987, 1009 (1010ff.).

64 Spindler, NJW 2004, 3145 (3147); Droste, CCZ 2015, 105 (108).

65 Siehe näher Spindler, NJW 2004, 3145 (3147).
Unfallschäden am autonom gesteuerten Fahrzeug selbst, die durch die Fehlsteuerung des Autopiloten verursacht wurden, da das Steuerungssystem ein funktionell abgrenzbares Produktteil ist, welches geeignet ist, das im Übrigen fehlerfreie Produkt zu beschädigen bzw. zu zerstören. ${ }^{66}$

Sofern der Hersteller im Zuge der Produktbeobachtung Fehler des Autopiloten erkannt hat, ist er bei der Auslieferung neuer Steuerungssysteme selbstverständlich zur Behebung des Fehlers, ggf. auch zur verbesserten Instruktion der Nutzer verpflichtet. ${ }^{67}$ Ebenso bedarf es der Warnung der Nutzer jener Produkte, die bereits in den Verkehr gelangt sind. ${ }^{68}$ Umstritten ist demgegenüber, ob und in welchem Umfang ein Hersteller zur Gefahrenbeseitigung hinsichtlich bereits in Verkehr gebrachter Produkte verpflichtet ist. Angesichts des hohen Gefährdungspotentials im Straßenverkehr nicht nur für den Fahrer des autonomen Fahrzeugs, sondern auch für andere Verkehrsteilnehmer, liegt es nahe, über die Warnung hinausgehend auch eine zivilrechtliche Rückrufpflicht anzunehmen. ${ }^{69}$ Beim Verdacht fehlender Produktsicherheit besteht ferner nach $\S 26$ Abs. 4 Produktsicherheitsgesetz eine öffentlichrechtliche Befugnis des Kraftfahrt-Bundesamts, den Rückruf des betreffenden Fahrzeugtyps anzuordnen. ${ }^{70}$

Warnung und Rückruf allein sind für den Fahrzeugeigentümer freilich nur in Grenzen interessant, da sie ihm nur die Schadensprävention durch Nichtverwendung des Fahrzeugs ermöglichen. Der Eigentümer wird deshalb auf eine Fehlerbehebung im Wege des Updates der Steuerungssoftware dringen, um einen sicheren Einsatz des Fahrzeugs im Straßenverkehr zu gewährleisten. Dieses

66 Meyer/Harland, CR 2007, 589 (691f.); Spindler, CR 2015, 766 (768) m.w.N.; Siehe weiterführend zum »Weiterfresserschaden" im Rahmen der Produzentenhaftung Sprau in Palandt, § 823 Rn. 178 m.w. N. 67 Wagner in MüKo-BGB, § 823 Rn. 676.

68 BGH NJW 1981, 1603 (1604f.); BGH NJW 1994, 3349 (3351); BGH NJW-RR 1995, 342 (343); Wagner in: MüKO-BGB, § 823 Rn. 677; Spindler in: Bamberger/Roth BGB, § 823 Rn. 514.

69 Ob eine Verkehrssicherungspflicht zum Produktrückruf besteht und falls ja, unter welchen Voraussetzungen, ist lebhaft umstritten, siehe BGH NJW 2009, 080 (verneinend für einen Fall professioneller Produktnutzer) sowie Wagner in: MüKo-BGB, § 823 Rn. $677 \mathrm{ff}$. und Spindler, Bamberger/Roth, BGB, § 823 Rn. 516ff., jeweils mit umfassenden Nachweisen zum Streitstand.

70 Die Zuständigkeit des Kraftfahrt-Bundesamts für die Durchführung des Produktsicherheitsgesetzes im Hinblick auf Produkte, die dem StVG unterliegen, ergibt sich aus $\S 2$ Abs. 1 Nr. 5a KBAG. Zum Umfang des Ermessens einer Anordnung des Rückrufs bei ernstem Risiko für die Sicherheit und Gesundheit von Personen gemäß § 26 Abs. 4 ProdSG siehe ausführlich Schucht in: Klindt (Hsrg.), Produktsicherheitsgesetz, 2. Aufl., München 2015, § 26 ProdSG Rn. 256 ff. Befolgt der Adressat die Rückrufanordnung nicht, kommt eine zivilrechtliche Haftung aus $\S 823$ Abs. 2 in Betracht, vgl. zutreffend Wagner in: MüKo-BGB, § 823 Rn. 699. 
Äquivalenzinteresse an der Nutzung des gekauften Fahrzeugs ist jedoch nicht deliktsrechtlich, sondern lediglich vertragsrechtlich geschützt. ${ }^{71}$ Ein Anspruch auf kostenloses Update der Steuerungssoftware kann sich somit grundsätzlich nur aus Vertrag, nicht aus Delikt, ergeben. ${ }^{72}$ Dem wird freilich mit Recht entgegengehalten, dass die Kostentragung durch den Hersteller einen Anreiz für den Fahrzeugeigentümer setzt, den Fehler zeitnah beheben zu lassen, und hierdurch den Rechtsgüterschutz dritter Personen sicherstellt. ${ }^{73}$

Der Streit dürfte für autonome Steuerungssysteme keine hohe Relevanz entfalten, da vertragliche Vereinbarungen zum regelmäßigen System-Update ohnehin zu erwarten sind. Autonome Steuerungssysteme unterliegen einer stetigen Fortentwicklung, wobei insbesondere Sicherheitslücken stetig erkannt und behoben werden müssen. Insoweit bleibt abzuwarten, welche Remunerationsstrategien die Fahrzeughersteller beim Vertrieb autonom gesteuerter Fahrzeuge verfolgen. Denkbar ist eine periodische Zahlungspflicht des Nutzers für den Betrieb des autonomen Steuerungssystems ebenso wie eine Herstellergarantie für den lebenslangen Betrieb eines einmal ausgelieferten Steuerungssystems. Zu erwarten ist ferner, dass Fahrzeughersteller bzw. Betreiber bereits aus Imagegründen solche Fehler des Autopiloten, welche die Fahrsicherheit beeinträchtigen, ohne Existenz einer Rechtspflicht beheben werden.

\section{Haftung des Halters}

Die Haftung des Halters ist im Straf- und Zivilrecht unterschiedlich ausgestaltet: Während der Halter im Zivilrecht aufgrund seiner Verfügungsgewalt über das Fahrzeug umfassend in die Pflicht genommen wird, scheidet eine genuin strafrechtliche Halterhaftung aus. Wenn künftig die technische Verfügungsgewalt über das Auto beim Betrei-

71 BGH NJW 2009, 1080 (1081f.); OLG Frankfurt VersR 1991, 1184 (1185); Foerste, in: Foerste/v.Westphalen, Produkthaftung, § 24 Rn. 340 ff.; Spindler, NJW 2004, 3145 (3148); ders., CR 2015, 766 (770). 72 Siehe allgemein BGH NJW 2009, 1080 (1081f.) m.w.N; Foerste, in: Foerste/v.Westphalen, § 24 Rn. 340 ff., 361; speziell für Software Astheimer, PHI 1986, 56 (59).

73 Staudinger/Czaplinski, JA 2008, 401 (405f.), die sich für eine einzelfallabhängige Teilung der Kostenlast aussprechen; für eine Kostenübernahme des Herstellers ferner OLG Karlsruhe NJW-RR 1995, 594 (497); OLG München VersR 1992, 1135f.; Wagner in: MüKo-BGB, $\S 823$ Rn. 679; Gomille, JZ 2016, 75 (80f.); Orthwein/Obst, CR 2009, 1, 3. Für eine Herleitung aus $\S 1004$ BGB Spindler in: Bamberger/Roth, BGB, § 823 Rn. 519. ber liegt und der Halter an Einwirkungsmöglichkeiten auf ein Fahrzeug verliert, das sich gegebenenfalls beständig aus der Datencloud aktualisiert, zieht dies nach sich, die zivilrechtliche Halterhaftung zu überdenken, weil dadurch die normalerweise dem Halter zugeordnete Verfügungsgewalt eingeschränkt wird.

\section{Verschuldenshaftung}

Den Halter eines Fahrzeugs trifft heute eine straf- oder zivilrechtliche Verschuldenshaftung, wenn er Auto und/ oder System vorsätzlich oder fahrlässig nicht ausreichend instand gehalten hat ${ }^{74}$ oder aber andere Aufsichts- oder Verkehrssicherungspflichten nicht beachtet hat. ${ }^{75}$

Eine - über allgemeine Zurechnungsregeln hinausgehende - strafrechtliche Halterhaftung statuiert das deutsche Strafrecht nicht. Dass dies auch anders gelöst werden kann, zeigt ein Blick in das benachbarte Ausland. In der Schweiz etwa kennt man eine strafrechtliche ausgestaltete Halterhaftung, niedergelegt in Regelungen des Straßenverkehrsgesetzes (SVG) ${ }^{76}$ Die verschiedenen Formen der Halterhaftung erscheinen begründungsbedürftig, wenn ein Halter das Fahrzeug gar nicht mehr selbst auf seine Betriebssicherheit überprüfen oder überprüfen lassen kann, weil das Fahrzeug selbst laufend Updates vornimmt. Sinnvoll ist eine durch Verkehrssicherungspflichten begründete Haftung nur insoweit, als es weiter der Halter ist, der Fehlfunktionen wahrnehmen kann und Wartungen durchführen lässt. ${ }^{77}$ Das dürfte mit zunehmender Automatisierung immer weniger der Fall sein.

\section{Verschuldensunabhängige Haftung nach § 7 StVG}

Aus zivilrechtlicher Perspektive erübrigt sich aufgrund der Gefährdungshaftung des Kfz-Halters gemäß $§ 7 \mathrm{StVG}^{78}$ die

74 Siehe hierzu bereits die Ausführungen supra C.I. zur Verschuldenshaftung des Fahrers.

75 Vgl. Wohlers, Straßenverkehr 1/2015, S. 5 ff.; Janker/Burmann, in: Burmann/Heß/Hühnermann/ Jahnke/Janker (Hrsg.), Straßenverkehrsrecht, 24. Aufl., München 2016, Rn. $54 \mathrm{ff}$.

76 Nach Art. 93 Abs. 2 lit. b SVG macht sich der Halter etwa strafbar, wenn er duldet, dass jemand sein Fahrzeug fährt, obwohl es nicht betriebssicher ist. Vgl. a. Art. 95 Abs. 1 lit. e Straßenverkehrsgesetz (SVG, SR 741.01) 〈https://www.admin.ch〉; Wohlers, Straßenverkehr 1/2015, S. 5 ff. (7f.); Schenk, Basler Kommentar Straßenverkehrsgesetz, Rn. $18 \mathrm{ff}$. zu Art. 93 SVG.

77 Vgl. Wohlers, Straßenverkehr 1/2015, S. 5 ff. (7f.).

78 Grüneberg in: Berz/Burmann (Hrsg.), Handbuch des Straßenverkehrsrechts, 34. EL, München 2015, Kap. 4 A. Rn. 5. 
Frage nach einem Verschulden des Halters. Gelegentlich wird die These vertreten, auf die Haftung des Halters könne künftig verzichtet werden. ${ }^{79}$ Ersatzweise wird eine Eigenhaftung des Fahrzeugs als intelligentem Agenten erwogen, unterstützt durch eine spezifische neuartige Versicherung. ${ }^{80}$ Gegenüber der derzeitigen Halterhaftung und der korrespondierenden Pflichtversicherung wäre durch eine solche Konstruktion freilich nichts gewonnen: Selbst wenn man autonom gesteuerte Fahrzeuge als rechtsfähig anerkennen würde, wäre die Zuordnung von Vermögen an dieses Rechtssubjekt ökonomisch nicht sinnvoll, da das Vermögen nur als Haftungsmasse bzw. zur Deckung der Versicherungsprämie genutzt werden könnte und im Übrigen totes Kapital wäre. Außerdem müsste ein rechtsfähiges Auto bei der Abgabe von Willenserklärungen (Abschluss des Versicherungsvertrags, Zahlungsanweisungen etc.) von einer natürlichen Person vertreten werden. ${ }^{81}$ Die Rechtsfigur des Halters bliebe also zumindest als Vertreter des intelligenten Agenten weiterhin erforderlich.

Das Konzept der zivilrechtlichen Halterhaftung erweist sich grundsätzlich auch für das autonome Fahren als tragfähig. Die in $\S 7$ StVG geregelte Gefährdungshaftung beruht auf dem Gedanken sozialer Verantwortung für die durch den Betrieb eines Kraftfahrzeugs geschaffene Gefahrenquelle. ${ }^{82}$ Dieser Haftungsgrund umfasst sowohl Risiken, die aus dem mechanischen Betrieb des Fahrzeugs resultieren, als auch Risiken, die aus der Steuerung des Fahrzeugs folgen (unabhängig davon, ob das Fahrzeug manuell oder autonom gesteuert wird).$^{83}$ Die Haftung trifft den Halter des Fahrzeugs, weil dieser den Nutzen aus der Verwendung desselben zieht und die Fahrzeugnutzung als

79 Hilgendorf, zitiert aus Behrens, Strafrecht für Autos, 〈http://www. sueddeutsche.de/auto/autonomes-fahren-strafrecht-fuer-autos1.1941244> vom 22. April 2014.

80 In diese Richtung z.B. Hilgendorf, Können Roboter schuldhaft handeln?, in: Beck (Hrsg.), Jenseits von Mensch und Maschine, Baden-Baden 2012, S. 127 f.; Beck, Brauchen wir ein Roboterrecht?, in: Japanisch-Deutsches Zentrum (Hrsg.), Mensch-Roboter-Interaktionen aus interkultureller Perspektive, Berlin 2012, S. 129f.; Matthias, Automaten als Träger von Rechten, Berlin 2008, S. 244; sowie Sartor, Agents in Cyber law, in: Proceedings of the Workshop on the Law of Electronic Agents, CIRSFID (LEA02) Gevenini, 2002, S. 7; siehe ferner Lutz, NJW 2015, 119, 121, der vorschlägt, autonome Fahrzeuge ausschließlich im Paket mit einer speziellen Haftpflichtversicherung anzubieten. Allgemein zur möglichen Rechtssubjektivität von Maschinen Kersten, JZ 2015, 1 (6f.).

81 Spindler, CR 2015, 766 (774f.).

82 König in: Hentschel/König/Dauer (Hrsg.), Straßenverkehrsrecht, 43. Aufl., München 2015, § 7 StVG Rn. 1; BGH VersR 2005, 992.

83 Walter in: Gsell/Krüger/Lorenz/Mayer (Hsrg.), BeckOK StVG § 7 Rn. $83 \mathrm{ff}$.
Gefahrenquelle kontrollieren kann. ${ }^{84}$ Beide Aspekte werden durch das autonome Fahren nicht berührt. ${ }^{85}$ Denn das von der Halterhaftung heute abgedeckte Spektrum wird auch künftig nicht vollständig durch die Produzentenhaftung des Herstellers bzw. Betreibers erfasst, selbst wenn der Betreiber die technische Verfügungsgewalt über das Steuerungssystem besitzt. Es wird weiterhin Risiken geben, für die Hersteller und Betreiber keine Verantwortung tragen, z.B. den Verschleiß und die mangelnde Wartung der physischen Fahrzeugkomponenten sowie manuelle Steuerungsfehler eines vom Autopiloten übernehmenden menschlichen Fahrers.

Die Haftung des Halters beugt schließlich Beweisproblemen des Geschädigten vor. Der Geschädigte kann den Halter in Anspruch nehmen, ohne den Beweis für die konkrete Unfallursache führen $\mathrm{zu}$ müssen. ${ }^{86}$ Eine solche $\mathrm{Zu}$ weisung des Beweisrisikos ist sachgerecht, da der Halter mit dem Betrieb des autonom steuerbaren Fahrzeugs auch die Gefahr geschaffen hat, dass sich die Schadensursache (mechanischer Defekt, manuelle oder autonome Steuerung) im Prozess mit dem Geschädigten nicht klären lässt.

Es mag jedoch Bereiche geben, in denen neu zu definieren ist, wann eine Halterhaftung eingreift und wann nicht. Ein nennenswerter Aspekt ist der unautorisierte Eingriff in den Autopiloten, insbesondere aufgrund eines Hackerangriffs. Eine vergleichbare Konstellation regelt heute $\S 7$ Abs. 3 S. 1 StVG für Schwarzfahrten: Ein schwarzfahrender Dritter haftet bei Nutzung des Fahrzeugs ohne Wissen und Willen des Halters anstelle des Halters, d.h. in gleichem Umfang wie dieser. Die Haftung des Kfz-Halters ist ausgeschlossen, wenn der Dritte das Fahrzeug ohne sein Wissen und Wollen benutzt und der Halter die Benutzung des Fahrzeugs nicht durch sein Verschulden ermöglicht hat. Nach der Rechtsprechung liegt eine "Benutzung " des Fahrzeugs durch denjenigen vor, der sich das Fahrzeug unter Verwendung der motorischen Kraft dienstbar macht und dadurch die Verfügungsgewalt wie ein Halter ausübt. ${ }^{87}$

Für Fremdsteuerungen des Autopiloten bietet sich de lege ferenda folgende Differenzierung an: Eine Benutzung des Fahrzeugs, die zur Haftung des Dritten neben dem

84 Grüneberg in: Berz/Burmann (Hrsg.), Handbuch des Straßenverkehrsrechts, 34. EL, München 2015, Kap. 4 A. Rn. 16.

85 Fleck/Thomas,NJOZ 2015, 1393, 1394; Riehm, ITRB 2014, 113, 114; Spindler, CR 2015, 766 (773f.).

86 Grüneberg in: Berz/Burmann (Hrsg.), Handbuch des Straßenverkehrsrechts, 34. EL, München 2015, Kap. 4 A. Rn. 72 f.

87 BGH NJW 1957, 500 (501ff.); König in: Hentschel/König/Dauer (Hrsg.), Straßenverkehrsrecht, 43. Aufl., München 2015, § 7 StVG Rn. 52. 
Halter führt, liegt bereits dann vor, wenn der Dritte die technische Verfügungsgewalt über das Fahrzeug erlangt, d.h. das Fahrzeug fremdsteuert, obgleich eine physische Verfügungsgewalt beim Halter verbleibt. Neben dem Hacker haftet in dieser Situation weiterhin der Halter, weil sich durch die Fremdsteuerung das typische Betriebsrisiko eines autonom gesteuerten Fahrzeugs realisiert. Demgegenüber sollte wie nach geltendem Recht die Halterhaftung ausgeschlossen sein, wenn der Dritte auch die physische Verfügungsgewalt über das Fahrzeug ausübt und der Halter die Fremdnutzung nicht durch sein Verschulden ermöglicht hat. In beiden Konstellationen haftet zudem der Hersteller bzw. der Betreiber, wenn das Eingreifen des Dritten durch einen Produktfehler ermöglicht wurde (supra C.II.2) d).

\section{Praktische Durchsetzung der Haftung}

Die praktische Durchsetzung straf- wie zivilrechtlicher Haftung steht und fällt mit den Möglichkeiten der Beweisführung im Prozess. Theoretisch wird die Klärung der Verursachungszusammenhänge mit der Einführung autonomer Fahrzeuge einfacher, weil zahlreiche Daten über die Begleitumstände des Unfalls gespeichert werden.

\section{Strafrechtliche Verfolgung}

Praktisch stellt sich im Strafverfahren die Frage, wie die Staatsanwaltschaft in einer Gemengelage von Wissensund Interessensasymmetrien agieren sollte. Bei Ermittlungen wegen des Verdachts von Straftaten im Zusammenhang mit automatisiertem Fahren gelten die allgemeinen Grundsätze. Das Beweisverfahren im Strafprozess ist jedoch traditionell auf den Nachweis von Schuld in der realen Welt mit menschlichen Akteuren konzipiert, nicht für Schnittstellen menschlicher Handlungen mit Roboterreaktionen auf der Grundlage elektronischer Datenverarbeitung. ${ }^{88}$ Die Notwendigkeit einer Beweisführung durch forensische Sachverständige ist ein (generelles) Problem bei der Verfolgung sog. Computerstraftaten. Hinzu kommt beim automatisiert fahrenden Auto die Notwendigkeit einer Abgrenzung der verschiedenen Verantwortungsbereiche in Herstellung und Betrieb. Der Wunsch nach einer möglichst lückenlosen Dokumentation könnte die datenschutzrechtliche Problematik des automatisierten Fahrens noch einmal verschärfen. Damit Autofahrer eine

88 Vgl. dazu etwa: Zerbes/El-Ghazi, NStZ 2015, 425 ff.
Unschuldsbeteuerung, ein Staupilot habe plötzlich nicht mehr funktioniert und nicht ordnungsgemäß zurückgerufen, untermauern könnten, müssten sie möglicherweise mit der Installation einer Kamera einverstanden sein, die stets den Fahrersitz im Visier hat. Inwieweit kann auf eine ordnungsgemäße Dokumentation durch Steuerungssysteme vertraut werden? Angesicht der jüngsten Manipulationsskandale der Automobilindustrie dürfte einerseits ein gewisses Misstrauen vorhanden sein. Was ist andererseits, wenn über Schuld oder Unschuld nur entschieden werden kann, indem Produzent oder Betreiber ein berechtigtes Interesse an der Wahrung von Betriebsgeheimnissen aufgeben und ihr System in einer öffentlichen Hauptverhandlung präsentieren müssen?

\section{Zivilrechtliche Beweislast}

Im Zivilrecht sind die Beweisfragen anders gelagert: Bei der Halterhaftung muss der Geschädigte lediglich die Ursächlichkeit des Kfz-Betriebs für den Unfall nachweisen, der Nachweis des Verschuldens von Halter oder Fahrer ist für die Gefährdungshaftung gemäß $\S 7$ Abs. 1 StVG nicht erforderlich.

Weitergehend ist die Beweislast des Geschädigten im Rahmen der Produzentenhaftung: Nach allgemeinen Beweisgrundsätzen liegt die Beweislast für das Vorliegen eines Produktfehlers und dessen Ursächlichkeit für die Rechtsgutsverletzung beim Anspruchsteller. Da der Geschädigte keinen Einblick in die internen Organisationsabläufe des Herstellers hat, kommt die Rechtsprechung dem Geschädigten bei der Produzentenhaftung mit einer Beweislastumkehr hinsichtlich des Verschuldens entgegen, d.h. der Hersteller muss das Einhalten der gebotenen inneren wie äußeren Sorgfalt nachweisen. ${ }^{89}$ Diese Beweislastverteilung dürfte den Bedürfnissen des Geschädigten bei Verkehrsunfällen mit autonom gesteuerten Fahrzeugen allerdings kaum gerecht werden..$^{90}$ Denn ebenso wie der Geschädigte keinen Einblick in die Interna des Herstellers hat, wird er regelmäßig keinen Einblick in die Funktionsweise des Steuerungsmechanismus haben. Es ist für den Geschädigten schwierig, zu beurteilen, ob ein verkehrswidriges Verhalten des autonom gesteuerten Fahrzeugs auf einem bereits bei Inverkehrbringen des Fahrzeugs vorhandenen Programmierfehler, auf fehlerhaften Updates, unzureichendem Datenmaterial, Versagen

89 Näher mit zahlreichen Nachweisen Wagner in: MüKo-BGB, § 823 Rn. 618, 623, $684 \mathrm{f}$.

90 Lutz/Tang/Lienkamp, NZV 2013, 57 (61); a.A. Gomille, JZ 2016, 75 (78). 
mechanischer Teile oder auf der Verschmutzung eines Sensors beruht.

Diesen Beweisschwierigkeiten kann auf dreierlei Wege begegnet werden: Erstens durch die Vermutung eines bereits bei Inverkehrbringen vorliegenden Produktfehlers, sofern das autonom gesteuerte Fahrzeug gegen Verkehrsregeln verstoßen hat; ${ }^{91}$ zweitens durch die Annahme einer Dokumentationspflicht des Herstellers bezüglich aller unfallrelevanter Daten des Steuerungssystems im Fahrdatenspeicher und eines gesetzlich geregelten Zugriffsrechts ${ }^{92}$ und drittens durch Einführung eines Auskunftsanspruchs des Geschädigten gegenüber dem Hersteller und/oder Betreiber. $^{93}$

\section{Rechtspolitische Bewertung}

Vor welche rechtpolitischen Aufgaben stellt uns damit die »Kutsche ohne Kutscher«?

\section{Strafrecht}

Aus strafrechtlicher Sicht ist zu entscheiden, ob und wenn ja wie Schuld nach tradierten Grundsätzen zugewiesen werden kann, wenn ein automatisiert fahrendes Auto einen Menschen überrollt. Die in den Betrieb dieses Fahrzeugs involvierten Menschen haften nach den allgemeinen Grundsätzen, wenn sie vorsätzlich oder fahrlässig für den Schadenseintritt verantwortlich sind. ${ }^{94}$ Vorsatzhaftung dürfte ein rarer Ausnahmefall sein. Beim Fahrlässigkeitsvorwurf stellen sich grundsätzliche Fragen: Sollen Fahrer, Halter, Produzent oder Betreiber das Risiko des algorithmisch getriebenen Automatismus tragen? Oder ist automatisiertes Fahren nach Zulassung eines Betriebssystems

91 So i.E. Gomille, JZ 2016, 75 (78), der von einem Anscheinsbeweis ausgeht. Siehe als potentielles gesetzliches Vorbild § 34 GenTG.

92 Vogt, NVZ 2003, 153 (160); Meyer/Harland, CR 2007, 689 (692); Lutz, NJW 2015, 119 (120); Spindler, CR 2015, 766 (772). Siehe zu vergleichbaren ärztlichen Dokumentationspflichten im Rahmen einer medizinischen Heilbehandlung $\S 630$ h Abs. 3 S. 2 und BGH NJW 1987, 1482 (1483); BGH NJW 1985, 2193 (2194). Bei den von der Rechtsprechung im Rahmen der Produzentenhaftung teilweise angenommenen "Befundsicherungspflichten « handelt es sich indes der Sache nach um Qualitätskontrollpflichten, vgl. die zutreffende Kritik von Wagner in: MüKo-BGB, § 823 Rn. 689 m.w. N. Zur potentiellen Übertragung auf Roboter siehe Spindler, CR 2015, 766 (772).

93 Als potentielles Vorbild siehe § 84a AMG.

94 Gless/Weigend, ZStW 2014, S. 588 ff.; Beck, Dealing with the diffusion of legal responsibility: the case of robotics, in: Battaglia/Mukerji/ Nida-Rümelin/Bisol (Hrsg.), Rethinking Responsibility in Science and Technology, Pisa 2014, S. 167 ff. (177 ff.). ein grundsätzlich erlaubtes Risiko? Bedarf es einer spezifischen Formulierung von Sorgfaltspflichten, weil Menschen über intelligente Systeme - anders als über andere Produkte - keine perfekte Kontrolle haben können?

Es ist durchaus denkbar, dass in fernerer Zukunft, wenn die »Kutsche ohne Kutscher« Normalität auf unseren Straßen ist, deren seltene Fehlfunktion als allgemeines Lebensrisiko akzeptiert wird, so wie wir heute bereit sind, einen für Menschen unvermeidlichen Verkehrsunfall als Unglück und nicht als Unrecht zu akzeptieren. Heute aber würden wir sicherlich die Menschen hinter der Maschine nicht einfach von strafrechtlicher Haftung freistellen, auch wenn es sehr gute Gründe gibt, das Strafrecht in dem Maße zurückzunehmen, in dem die Allgemeinheit automatisiertes Fahren trotz der damit verbundenen Risiken wegen des erhofften sozialen Nutzens begrüßt. Rechtspolitisch bedarf es einer Diskussion darüber, ob die Gesellschaft grundsätzlich bereit ist, zu akzeptieren, dass selbst nach tödlichen Autounfällen Strafverfolgung eine echte Ausnahme würde. Denn wenn den involvierten Menschen kein Vorwurf mehr gemacht werden kann, bliebe nur noch das Auto oder sein Betriebssystem als Adressat des strafrechtlichen Vorwurfs, und diese verfügen weder über eine - für einen Menschen nachvollziehbare - Strafempfindlichkeit noch würden sie den mit der Bestrafung verbundenen ethischen Vorwurf verstehen. Anders als bei der Strafbarkeit juristischer Personen ließe sich eine Bestrafung von Robotern auch nicht als mediatisierte Bestrafung der im Hintergrund stehenden natürlichen Personen für ein "Organisationsverschulden « interpretieren. ${ }^{95}$ Eine solche Grundsatzdiskussion wäre eine seltene Gelegenheit, sich wieder einmal $\mathrm{zu}$ vergegenwärtigen, was genau das Menschliche am Menschen ist, das ihn zum vernünftigen Adressaten eines strafrechtlichen Vorwurfs macht. ${ }^{96}$

\section{Zivilrecht}

Die Betrachtung bestehender Haftungskonzepte im Zivilrecht hat gezeigt, dass Instrumente für eine sachgerechte Haftungsverteilung grundsätzlich vorhanden sind. Die Gefährdungshaftung des Kfz-Halters ist auch nach Einführung autonom fahrenden Autos weiterhin adäquat. Zwar mag der Halter aufgrund der technischen Verfügungsgewalt des Betreibers künftig weniger Einwirkungsmöglichkeiten auf sein Fahrzeug haben. Dennoch zieht der Halter weiterhin die Verwendungsnutzungen aus dem

95 Gless/Weigend, ZStW 2014, S. $588 \mathrm{ff}$. 96 Gless/Weigend, ZStW 2014, S. $588 \mathrm{ff}$. 
Auto und kann jedenfalls die physische Fahrzeugnutzung und den physischen Zustand des Fahrzeugs als Gefahrenquelle kontrollieren. Schließlich ist der Fahrzeughalter ein für den Geschädigten einfach zu identifizierendes Haftungssubjekt.

Auch die Haftung des Fahrzeugherstellers sowie des Betreibers kann nach den Grundsätzen der Produzentenhaftung de lege lata zumindest prinzipiell begründet werden. Hier stellen sich drei Problemkreise: Erstens, wie gelingt dem Geschädigten bzw. der vom Geschädigten in Anspruch genommenen Versicherung des Halters der Nachweis, dass ein Fahrfehler des Autos auf einen Produktfehler zurückzuführen ist? Zweitens, sollte der Betreiber auch für die von ihm beauftragten Drittunternehmen (Datenlieferanten, Programm-Zulieferer) strikt einstehen müssen, d.h. sollte die derzeit im Rahmen der Produzentenhaftung bestehende Entlastungsmöglichkeit für den Verantwortungsbereich des Zulieferers abgeschafft werden? Drittens, ist es angesichts der technischen Verfügungsgewalt des Betreibers sachgerecht, wenn der Geschädigte - wie nach derzeitiger Rechtslage - zunächst vom Halter bzw. dessen Versicherung Ersatz begehrt und die Versicherung sodann den Betreiber in Regress nimmt?

Aufgrund der Komplexität autonomer Fahrprozesse wird es dem Geschädigten bzw. dem Halter oftmals schwerfallen, einen Produktfehler nachzuweisen. Sinnvoll wäre deshalb eine gesetzliche Vermutung, dass ein verkehrswidriges Verhalten des autonomen Fahrzeugs auf einen Produktfehler des Steuerungssystems zurückzuführen ist. Auch eine Gefährdungshaftung des Betreibers für sämtliche Produktfehler erscheint plausibel, so dass der Betreiber auch für Fehler der von ihm beauftragten Programmierfirmen und Datenlieferanten einzustehen hätte. Als Vorbild könnte das Produkthaftungsgesetz dienen, ${ }^{97}$ dessen Haftungskonzept sich derzeit auf Produktfehler beweglicher Sachen beschränkt. Die dritte Frage lässt sich auf Basis unseres derzeitigen Kenntnisstands nicht befriedigend beantworten. Wenn die Visionen der Branche Wirklichkeit werden und der Verkehr künftig von intelligenten, vernetzten und angriffssicher gestalteten Fahrzeugen reibungslos selbst geregelt wird, so sind Verkehrsunfälle eventuell nur noch dem durch Verschleiß verursachten Versagen mechanischer Fahrzeugteile zuzuschreiben. Dann erscheint es sachgerecht, wenn Geschädigte weiterhin aufgrund der Gefährdungshaftung des $\S 7$ StVG vom Halter Ersatz beanspruchen und jener gegebenenfalls Rückgriff beim Hersteller und/oder Betreiber nimmt. Erweisen sich hingegen Sys-

$97 \S 4$ ProdHaftG. temfehler als Hauptursachen künftiger Verkehrsunfälle, könnte es durchaus angemessen sein, eine Gefährdungshaftung des Betreibers für sämtliche Fahrfehler des Fahrzeugs einzuführen. Der Betreiber müsste sodann Regress beim Halter nehmen, wenn sich ein anderes Betriebsrisiko als eine Fehlfunktion des Steuerungssystems verwirklicht hätte.

\section{E. Das kalkulierbare Dilemma}

Dass das autonom fahrende Auto sowohl aus straf- als auch aus zivilrechtlicher Sicht alte Grundsatzfragen in neuem Licht aufwirft, zeigt sich wenn man die altbekannten Dilemmata-Fälle auf die »Kutsche ohne Kutscher« anwendet. Wenn sich heute ein menschlicher Fahrer mit einem plötzlich auftauchenden, brennenden Unfallfahrzeug konfrontiert sieht und entscheidet sich nicht selbst zu opfern, sondern in eine Personengruppe zu fahren, die sich am Autobahnrand in Sicherheit gebracht hat, dann stellt er sein Leben über das anderer - und begeht Unrecht. Den Fahrer trifft gleichwohl nicht unbedingt ein strafrechtlicher Schuldvorwurf, denn das Recht verlangt vom Menschen nicht das Übermenschliche. ${ }^{98}$

Würde ein Auto-Pilot mit dieser Entscheidung konfrontiert, stellt sich die Lage anders dar: Ein Auto darf nicht die eigene Unversehrtheit über Menschenleben stellen. Es müsste in das brennende Hindernis fahren - wären da nicht die Insassen. Deren Leben darf »das Auto« aber von Rechts wegen - nur wie Rechtsgüter unbeteiligter Dritter mit den Rechtsgütern der am Rand stehenden Personen abwägen. Das entspricht auf den ersten Blick dem traditionellen "Weichensteller«-Dilemma. Nach in Deutschland herrschender Meinung muss man in diesen Fällen einfach dem Geschehen seinen Lauf lassen, um sich nicht schuldig zu machen. ${ }^{99}$

98 Stratenwerth/Kuhlen, Strafrecht Allgemeiner Teil, 6. Aufl., München 2011, § 10 Rn. 104 ff.; Frister, Strafrecht Allgemeiner Teil, 7. Aufl., München 2015, S. $264 \mathrm{ff} . ;$ Roxin, Strafrecht Allgemeiner Teil, Band I, 4. Aufl., München 2006, § 22 Rn. 1ff.; vgl. auch Stratenwerth, Schweizerisches Strafrecht, Allgemeiner Teil I, 4. Aufl., Bern 2011, § 11 Rn. 65 ff.; Seelmann, Basler Kommentar Strafrecht I, Rn. 1ff. zu Art. 18 StGB.

99 Roxin, Strafrecht Allgemeiner Teil, Band I, 4. Aufl., München 2006, § 16 Rn. 33ff., 115ff.; Jescheck/Weigend, Lehrbuch des Strafrechts, Allgemeiner Teil, 5. Aufl., Berlin 1996, S. 365 ff., 501ff.; Perron, Kommentar Strafgesetzbuch, 29. Aufl., München 2014, § 34 Rn. 23f.; Lenckner/Sternberg-Lieben, Kommentar Strafgesetzbuch, Vor §§ $32 \mathrm{ff}$. Rn. 115ff.; vgl. auch Stratenwerth, Schweizerisches Strafrecht, Allgemeiner Teil I, 4. Aufl., Bern 2011, § 10 Rn. 45; § 11 Rn. 85. 
Diese Ansicht einmal dahingestellt, tut sich dahinter jetzt ein neues, kalkulierbares Dilemma auf. Eine Programmiererin kann und muss »das Geschehen in der Not« in aller Ruhe im Voraus bedenken und dafür Handlungsanweisungen geben. Aus ihrer Sicht gibt es keinen plötzlich »sich von selbst entfaltenden Verlauf«. Was soll sie tun? Darf sie sich »den menschlichem Überlebenswillen« der Passagiere zu eigen machen oder muss sie einen objektiv abwägenden, rationalen Ansatz verfolgen? Darf sie dem individuellen Autofahrer unterschiedliche Einstellungen als wählbare Optionen zur Verfügung stellen?

Für einen objektiv abwägenden, rationalen Ansatz spricht, dass Passagiere eines Roboterautos bereits Vorteile genießen. Warum sollten sie auch noch in einer Notsituation ihre technische Überlegenheit auf Kosten Unbeteiligter nutzen dürfen? Gleichzeitig muss sich ein Hersteller eines autonom fahrenden Autos fragen lassen, warum sich jemand ein solches Kfz zulegen sollte, wenn man sehenden Auges in Kauf nehmen müsste, sich in einer bedrohlichen Situation nicht mehr retten zu dürfen. Außerdem greift die Erwägung der technischen Überlegenheit nicht immer durch. So wäre etwa für bestimmte Situationen in Rechnung zu stellen, dass andere Menschen gegenüber den Passagieren eines autonom fahrenden Fahrzeugs unrecht handeln könnten; etwa wenn sie sich - um ein Unfallgeschehen live zu erleben - genau in den Bereich begeben, in den das selbstfahrende Auto ausweichen könnte. Angesichts der schwierigen Einzelfragen erschiene es vielleicht am Ende tatsächlich besser, jeweils betroffene Menschenleben zu zählen, ${ }^{100}$ was aber aus deutscher Sicht kaum denkbar ist. ${ }^{101}$ Denn jedenfalls in Deutschland wird diese Diskussion im Kontext der Abgrenzung zur menschenverachtenden Staatsräson des Nationalsozialismus geführt und berührt ein Tabu des heutigen Rechtskonsenses. ${ }^{102}$ Möglicherweise wird die Notwendigkeit, eine Lösung für das vorprogrammierte Dilemma zu finden, die

100 Foot, Killing and Letting Die, in: Foot (Hrsg.), Moral dilemmas and other topics in moral philosophy, Oxford 2002, S. 78ff.; Edmonds, Would You Kill the Fat Man? Trolley Problem and What Your Answer Tells Us about Right and Wrong, Princeton 2014; vgl. auch Hilgendorf, Recht und autonome Maschinen - ein Problemaufriß, in: Das Recht vor den Herausforderungen der modernen Technik, Robotik und Recht Band 4, Baden-Baden 2015, S. 11 ff. (20 ff.).

101 Vgl. etwa BVerfGE, Urteil vom 15. Februar 2006 - 1 BvR 357/05 (= BVerfG 115, 118), Rn. 119 ff.; Kindhäuser/Neumann/Paeffgen, NK StGB, 4. Aufl., Baden-Baden 2013, Vor $\S 35$ Rn. 54 ff.; Roxin, Strafrecht Allgemeiner Teil, Band I, 4. Aufl., München 2006, S. 738 ff.; Hilgendorf, Recht und autonome Maschinen - ein Problemaufriß, in: Das Recht vor den Herausforderungen der modernen Technik, Robotik und Recht Band 4, Baden-Baden 2015, S. $11 \mathrm{ff}$.

102 Vgl. etwa Hartleb, NJW 2005, 1397 ff.
Debatte auf eine rationalere, eher utilitaristischere Grundlage stellen. ${ }^{103}$

Die Dilemma-Situationen spitzen Grundfragen einer digitalisierten Lebenswelt zu, die sich in anderen Bereichen ebenfalls stellen: Die Entscheidung von Interessenkonflikten zwischen Individualbedürfnissen und gesamtgesellschaftlichen Anliegen wird vorprogrammiert und nimmt unter Umständen dem Einzelnen die Möglichkeit, eigene Interessen, sogar das eigene Überlebensinteresse gegen eine kalkulierte Verhaltensregel durchzusetzen. ${ }^{104}$

\section{F. Blick nach vorne}

Wenn wir - in einer ferneren oder näheren Zukunft - nach einem Auffahrknall im Stop-and-go-Verkehr nicht mehr den Fahrer des Auffahrwagens verwünschen, sondern direkt die Marke des aufgefahrenen Wagens oder des darin verwendeten Betriebssystems ins Visier nehmen oder gar spontan einen möglichen Angriff von außen bedenken, dann hätte die prognostizierte »Revolution der Automobilität« unser Unterbewusstsein erreicht. Wir hätten dann verinnerlicht, dass der Gewinn an mehr Verkehrssicherheit und Bequemlichkeit maßgeblich den stets auf Weiterentwicklung und Überwachung ihrer Produkte bedachten Herstellern zu verdanken ist, dass aber Fehlfunktionen nicht gänzlich auszuschließen sind und unter anderem durch eine Vernetzung der Verkehrsteilnehmer erkauft werden müssen, die neue Sicherheitsfragen und Datenschutzprobleme mit sich bringen.

Die Zeit der »Kutsche ohne Kutscher « scheint angebrochen, eine Rückkehr zum Pferd ausgeschlossen. Fahrerlose Transportmittel dürften zukünftig selbstverständlich zum Straßenverkehr gehören. Ob dann (nach heutigen Prognosen nur noch sehr selten vorkommende) Unfälle als »allgemeines Lebensrisiko«, ähnlich dem von einem Baum herabfallenden Ast, akzeptiert werden, dürfte einerseits von der Unfallhäufigkeit abhängen. Andererseits dürfte entscheidend sein, ob es gelingt, die neue Verantwortungsverteilung normativ zu fassen und real durchzusetzen. Mit seiner sichtbaren Pionierrolle trägt automatisiertes Fahren die Bürde solcher teilweise dramatisierter Fragestellungen. Es hat aber auch alle Chancen eines echten Vorreiters.

103 Siehe auch Wohlers, Basler Juristische Mitteilungen 2/2016 (im Erscheinen).

104 Eine empirische Momentaufnahme der Widersprüche zwischen abstrakter und konkreter Bewertung, Moral und Kaufentscheidung, zeigen Bonnefon/Shariff/Rahwa, The social dilemma of autonomous vehicles, 352 Science (2016), S. 1573-1576; siehe auch «http://moralmachine.mit.edu . 